\title{
Numerical studies to determine spatial deviations of a workpiece that occur when machining on CNC machines
}

\author{
Roman Yu. Nekrasov ${ }^{1,}$, Igor V. Solovyov ${ }^{1}$, Alexander I. Starikov ${ }^{1}$, Yulia A. Tempel $^{1}$, and Olga A. Tempel ${ }^{1}$ \\ ${ }^{1}$ Industrial University of Tyumen, 38 Volodarskogo st., Tyumen, Russia, 625000
}

\begin{abstract}
The article discusses the problem of increasing the accuracy of machining workpieces on machines with computer numerical control. Within the framework of the problem identified, the role of the finite element method for solving it is considered. Moreover, numerical simulation is primarily used for receiving estimates of operational and technological characteristics of the developed product, and in the present work we propose to use a method of the finite element analysis as a tool for ensuring the accuracy of shaping the surface of a workpiece in process systems for determining the deformation of the workpiece under the action of cutting forces and transformation of its geometric shape prior to direct machining based on identified deviations. That is, the possibility of applying the finite element method in the development of control programs for details such as bodies of revolution is considered.
\end{abstract}

\section{Introduction}

At present, due to sharp competition in the domestic and global markets, machine-building products are subject to strict requirements, provision of which is the main goal of enterprises of the industry.

High productivity and flexibility of production, and, as a result, reduced cost of finished products, allows one to provide machine tools with computer numerical control (CNC) which has wide application in modern machine building.

However, despite the fact that equipment equipped with CNC systems has high discreteness of movements, functions of adjusting the trajectory of the cutting tool, taking into account the wear of the cutting edge and other technical possibilities, the accuracy of processing does not always correspond to the established requirements. Therefore, increasing the accuracy of machining at this time is one of the most pressing issues in engineering technology and cutting theory, and one of the priority areas of machine-building production.

Therefore, many researchers and scientists in this field seek to solve this problem in various ways, but pursue the same goal - the output of a high probability of products that meet the established requirements, as well as preservation of material resources, energy, labor and time in the production of high-precision parts of modern production.

Computer correction of movements of the executive working bodies of the machine tool, development and investigation of intelligent control systems, automatic control of geometric accuracy and regulation of power parameters during processing are among the significant areas in the research of authors in the field of improving the accuracy and quality of processing. But, since a lot of factors influence the machining process, the urgent task for today is to replenish the already existing knowledge base in the area under consideration and increase the efficiency of modern machine-building production.

In addition, requirements for designing in machine building are constantly increasing, in connection with the introduction of new technological processes, modes, materials in the manufacture of parts with high requirements for dimensional accuracy and service life, so the use of numerical simulation is the most profitable.

One of the methods of numerical simulation which is widely applied in the field of engineering analysis is the finite element method (FEM). It serves as a more perfect instrument for making calculations when solving problems of ensuring the reliability of projects in the field of machine building.

In connection with the foregoing, the purpose of the study is: the use of numerical research methods and consideration of the possibility of their application in the development of control programs for processing parts such as bodies of revolution in order to improve the accuracy of their shaping.

\section{Materials and Methods}

To accomplish the research tasks, theoretical foundations and scientific positions formulated in the works of Russian scientists in the field of engineering technology, automation and control of technological processes, cutting metals were used. When performing the work, methods of calculus mathematics, mathematical modeling, matrix analysis and statistical processing of results of experiments were used. For the finite element simulation of the effect of cutting force components on

Corresponding author: nekrasovri@,tyuiu.ru 
the object of investigation, the software product SolidWorks Simulation was used.

\section{Results and Discussion}

Mathematical modeling and numerical studies of various phenomena and processes are becoming increasingly important. This is due to the ability to view complex objects in different environments, as well as the costs of conducting full-scale experiments.

When turning parts, the tool performs the function of shaping, that is, it must ensure that surfaces of specified sizes and shapes are obtained. The solution of such problems is largely determined by the kinematics of the cutting process. The kinematics of shaping by turning is considered as a combination of four components: shape of the resulting surface of the part; shape of the cutting tool producing surface; shaping movements of the cutter relative to the workpiece; position of the cutter and the workpiece relative to each other.

In order to provide the possibility of assigning the necessary technological conditions for the forming of a part within the specified tolerance limits, a number of the following tasks are realized: building a mathematical model for shaping the surface of a part with the help of an edge of a cutting tool, as well as carrying out numerical studies using the finite element method to determine spatial deviations of a workpiece that arise during machining on $\mathrm{CNC}$ machines under the action of cutting force components and the possibility of their compensation.

In the process of machining a workpiece, the machine is subjected to intensive impact from a whole range of operational loads of a random nature, as a result of which the trajectories of the forming elements change widely, which in turn leads to a change in the shape of the part and its accuracy parameters. And, as you know, increasing the accuracy of manufacturing parts increases the service life of machines and equipment. If accuracy of parts that make up the machine is inadequate, the quality of its functioning will be low and in the course of work significant dynamic loads can occur that lead to accelerated deterioration of the equipment and its destruction.

Many Russian authors [eg, 1,2,3,4] consider the influence of processing errors caused by cutting forces, thermal phenomena and wear of the cutting tool to be critical. They lead to a change in the shape and spatial position of both the machine executive organs and the workpiece.

Since cutting is a multifactor process and its complete analytical description is practically impossible at present $[4,5]$, in this paper a narrow problem was solved aimed at modeling the transformation of the part model under the influence of elastic force deformations during the turning process and assessing their effect on the dimensional accuracy of the finished product. The calculation was made using a number of the above assumptions: calculations did not take into account thermal deformations that occur in the cutting area; operating conditions of the $\mathrm{CNC}$ machine tool, its life and accuracy were also not taken into account.

In connection with the foregoing, to determine specific values of spatial deviations of the real profile of the part from the nominal during processing under the action of elastic deformations and subsequently with their account to model the actual CAD model of the shaft, it is necessary to perform numerical simulation.

One of the methods of numerical simulation which is widely applied in the field of engineering analysis is the finite element method (FEM). It serves as a more perfect instrument for making calculations when solving problems of ensuring the reliability of projects in the field of machine building. Unlimited possibilities for realization of computer models on the basis of FEM led to the development of new methods of mathematical physics to solve problems of dynamics, stability, physical and geometric nonlinearity [6]. The FEM module is a powerful tool for mathematical solution of the issues of durability and overall design reliability.

Since in the total volume of machining the share of manufacturing body parts and details like body of rotation is about $55 \%$, the shaft of steel grade Steel $40 \mathrm{X}$ (GOST 4543-71) served as the object of investigation. As an example, we consider a workpiece at turning, fixed in a lathe chuck without a rear center (Figure 1).

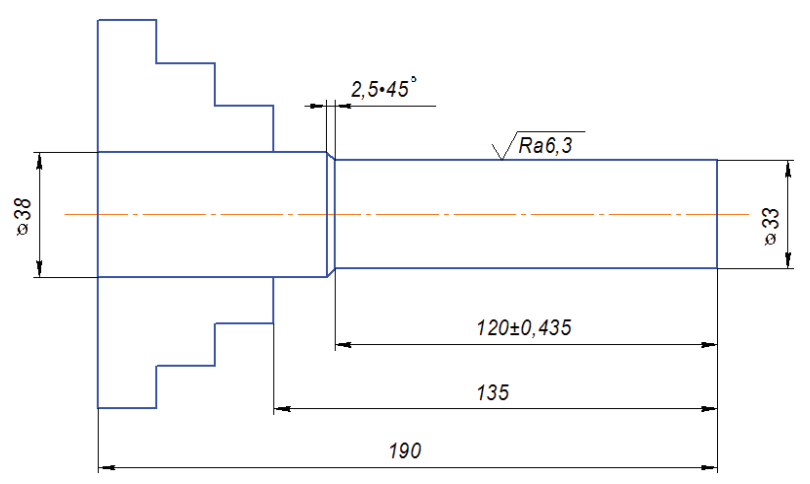

Fig. 1. Workpiece sketch.

For finite element analysis, calculating cutting force components that arise during the turning of a cylindrical surface of a piece with a diameter of $33 \mathrm{~mm}$ and a length of $120 \mathrm{~mm}$ with a single rough turning, with a cutting depth $\mathrm{t}=2.5 \mathrm{~mm}$, a cutting tool - a straight-turning tool T15K6 GOST 18878-73 with the size of the holder $16 \times$ 25 and the following geometric elements of the tool: plan relief angle $\varphi^{\circ}=45^{\circ}$; rake angle $\gamma^{\circ}=0^{\circ}$; pitch angle of the main blade $\lambda^{\circ}=0^{\circ}$, a computational model is created with the physical-mechanical properties of the selected material. Properties of the selected material are as follows: elastic modulus $\mathrm{E}=1.76 \cdot 10^{-5} \mathrm{MPa}$; Poisson's ratio $v=0.25$; density $\rho=7670 \mathrm{~kg} / \mathrm{m}^{3}$. The set load is the components of the cutting force $\mathrm{P}_{\mathrm{z}, \mathrm{y}, \mathrm{x}}=2798 / 1392 / 1392$ N.

The deformed body is divided into finite elements. As a result of the breakdown, a grid is created from the boundaries of the elements. The intersections of these boundaries form nodes. Additional node points can be created at the boundaries of and inside the elements. An ensemble of all finite elements and nodes is the basis of 
the finite element model of the deformed body. The discrete model should cover the area of the object under investigation fairly well.

The results of the finite element analysis show the change in the spatial shape of the object under study, the magnitude of the resulting displacements of which is shown in Figure 2, they are indicated in micrometers.

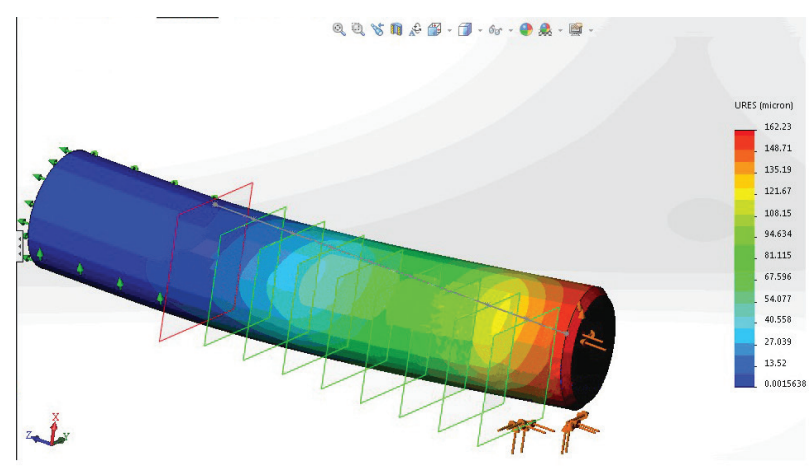

Fig. 2. Results of analysis of displacements of the finite element model.

As Figure 2 shows, after turning, the shape of the part is not cylindrical, but saddle-shaped as a result of deformation from the applied cutting forces.

As the designer provides the CNC programmer with the geometric model of the workpiece which is doomed in advance to the occurrence of errors related to the elastic force deformation of the process equipment, the solution to the problem lies in changing the geometric model of the workpiece by generating a control trajectory and creating a control program.

In connection with the above, based on the numerical simulation, the next step was the introduction of changes to the original geometric model recorded in the STEP format. To confirm the effectiveness of the proposed method for controlling the geometric accuracy of the workpiece on CNC machines, two batches of parts were tested, each of which consisted of five pieces processed by a straight-turning tool T15K6 GOST $18882-73$ with a holder size of $16 \times 25$ on the turning center SMTCL CAK50135. The first batch was processed without taking into account the arising errors; the second batch of parts was processed according to the program with a modified trajectory of the tool tip displacement on the basis of the transformed CAD model.

To assess reliability indexes according to the quality parameters of the manufactured products, depending on the type of technical system and the evaluation purposes, experimental-statistical methods were used. When comparing the results of measurements and determining the reliability of the technological operation when processing parts without changing the control program and processed according to the proposed method of geometrical accuracy control, it can be said that the efficiency of the method is confirmed by experiments and analysis of data obtained in the experiment. Based on the analysis of distribution of a random variable, there is a constant shift in the mathematical expectation in a batch of parts processed by a control program with a transformable CAD model. In addition, standard deviation is reduced by $40.9 \%$, and the range of variation - almost twofold.

\section{Conclusion}

In the modern scientific literature, much attention is paid to the quality and precision of metal working on machines with computer numerical control. In connection with this an urgent task was to identify the main trends and problems arising in this direction of the cognitive activity of the scientific community.

In the presented research, the following goal was achieved: increasing the dimensional accuracy of the shaping of parts such as bodies of revolution, the quality of their metalworking, the efficiency of using the production accuracy of technological equipment when turning on machines with computer numerical control.

To realize this goal, factors that affect the quality and accuracy of machining on CNC machines were analyzed. Based on this analysis, and also taking into account the fact that cutting is a rather complicated process, a narrow task was solved in the work to simulate transformations of the CAD model of the stepped shaft, considering error compensation that occurs during the action of the cutting force on the workpiece during turning.

An approach is proposed for determining elastic deformations of a workpiece under the influence of cutting forces and controlling the accuracy of machining on CNC machines using the finite element method.

Reliability of the obtained results is confirmed by numerical and physical experiments.

Reliability of the technological operation of machining of two batches of parts has been estimated using methods of statistical analysis. The first batch is parts manufactured according to the control program with the modified CAD model of the workpiece, and the second batch - without modification. Comparison of the results of assessment confirms the effectiveness of the proposed method for controlling the geometric accuracy of machining of shaft-like parts on CNC machines.

\section{References}

1. A.F. Lysenko, A.I. Izyumov, O.V. Goncharov, Bul. of Don State Tech. Univ., 14, No.3(78) 96-102 [Electronic resource] URL: http:// Elibrary.ru (2014)

2. R.Yu. Nekrasov, A.I. Starikov, I.V. Soloviev, O.V. Bekareva, Tech. and Mat., 4, 41-48, (2015)

3. I.A. Shchurov, I.S. Boldyrev, Bul. of South Ural State Univ., Mechanical Engineering, 12, 143-149, (2012)

4. A.S. Kuznetsov, A.A. Drozdov, Modern problems of science and education, 2, 165-170 [Electronic resource] URL: http:// Elibrary.ru (Penza, 2015)

5. I.V. Soloviev, Yu.A. Tempel, O.A. Tempel, IPDM2017 (St. Petersburg, 2017)

6. H.M. Muselemov, O.M. Ustarkhanov, I.A. Ferzaliev, Science in Figures, 1, 14-16, (2016) 
7. V.G. Shalamov, Mathematical modeling in cutting metals (Chelyabinsk, 2017)

8. A.A. Lasukov, V.V. Dureev, Mining Inf. and An. Bul., 2, 314-322 (2011)

9. A.S. Yanyushkin, S.O. Safonov, D.V. Lobanov, Perfection of technological processes of machinebuilding productions (Bratsk, 2006)

10. A.A. Lasukov, A.A. Mokhovikov, IFOST 2012 6357720, IOP Conf. Series: Mater. Sc. and Eng., 142 (2016)

11. U.S. Putilova, Yu.I. Nekrasov, A.A. Lasukov, Appl. Mech. and Mat., 682, 192-195, (2014) 